\title{
Polymer waveguide end facet roughness and optical input/output coupling loss for OPCB applications
}

\author{
Hadi Baghsiahi*a $^{\text {a }}$ Kai Wang ${ }^{\mathrm{b}}$, Richard Pitwon ${ }^{\mathrm{b}}$, David R. Selviah ${ }^{\mathrm{a}}$ \\ ${ }^{a}$ Department of Electronic and Electrical Engineering, University College London (UCL), \\ Torrington Place, London, WC1E 7J, UK, +44(0)2076794156 \\ h.baghsiahi@ee.ucl.ac.uk, d.selviah@ee.ucl.ac.uk \\ ${ }^{b}$ Xyratex Technology Ltd, 1000-40 Langstone Technology Park, Havant, Hampshire, PO9 1SA, UK \\ +44 (0)2392496715
}

\begin{abstract}
Electro-optical printed circuit board technology (EOCB) based on integrated planar polymer optical waveguides has been the subject of research and development for many years to provide a cost viable, fully integrated system embedded optical interconnect solution, however a number of constraints of this technology have yet to be overcome. Optical coupling loss at the input and output of the waveguides is one of the major issues and waveguide end facet roughness is one of the main sources of the coupling loss which is investigated in this paper.

The results of a comprehensive investigation of the end facet roughness of multimode polymer waveguides, fabricated on FR4 printed circuit boards, PCBs, and its effect of optical loss are presented theoretically and experimentally. The waveguide end facet roughness was measured using an atomic force microscope, AFM, when the waveguides were cut using a milling router with various numbers of cutting edges called flutes. The optimized cutting parameters are derived and the optical coupling loss, between the laser source and the waveguide, due to the different roughness magnitudes is measured by experiment for the first time. To improve the surface quality and decrease the waveguide optical loss, a new fabrication technique for reducing the end facet roughness after cutting is proposed and demonstrated. The insertion loss was reduced by $2.60 \mathrm{~dB} \pm 1.3 \mathrm{~dB}$ which is more than that achieved by other conventional methods such as index matching fluid.
\end{abstract}

Keywords: Optical waveguide, waveguide roughness, optical insertion loss, optical interconnect, OPCB, high data rate, multimode waveguide, microfabrication, microtooling, microengineering, nanoroughness, coupling loss.

\section{INTRODUCTION}

The demand for optical printed circuit board (OPCB) products is growing rapidly as the demand for high data rate communication is growing [1]. However, manufacturing cost and sufficient optical power transmission are two of the challenges for this approach [2]. New research reported in this paper aims to determine the best method of manufacturing, assembling and designing OPCBs. To develop low-cost manufacturing techniques for integrated OPCBs, the optical coupling efficiency between the waveguide and the laser source is very important as it affects the optical power budget and the bit error rate [3]. The optical coupling efficiency depends on the roughness of the end facet of the waveguide, the lateral and transverse alignment of the waveguide to the laser [4-6] and the refractive index difference between the medium of the laser and the core material. In the OPCB design developed at UCL, the daughter board is connected to the backplane by butt-coupling to the waveguide using the new UCL and Xyratex optical connector design [3]. Therefore, the backplane board needs to be cut to make places for the connector mounted on the daughterboard. Research by other groups uses edge connectors where optical connectors make contact with the OPCB only around its edges. In such an arrangement the board may be cut with a dicing saw. However, this limits the flexibility of layout possible and does not match the requirements for replacement of PCB backplanes in existing rack system configurations. The UCL and Xyratex mid-board connector offers layout flexibility and compatibility with rack system configurations but dicing saws cannot be used to cut out small apertures in the middle of the board. Figure 1 shows a picture of an OPCB developed at UCL and four places to be cut (white rectangular parts in the middle of the board).

Integrated Optics: Devices, Materials, and Technologies XVIII, edited by Jean Emmanuel Broquin,

Gualtiero Nunzi Conti, Proc. of SPIE Vol. 8988, 898804 - (c) 2014 SPIE

CCC code: $0277-786 \mathrm{X} / 14 / \$ 18 \cdot$ doi: $10.1117 / 12.2039785$ 
The polymer waveguide samples used in this research were made by photolithography and cut by two different methods for comparison: dicing saw and milling router. A milling router is used to cut optical polymer waveguide and the effect of this method on the waveguide surface profile is investigated in detail.

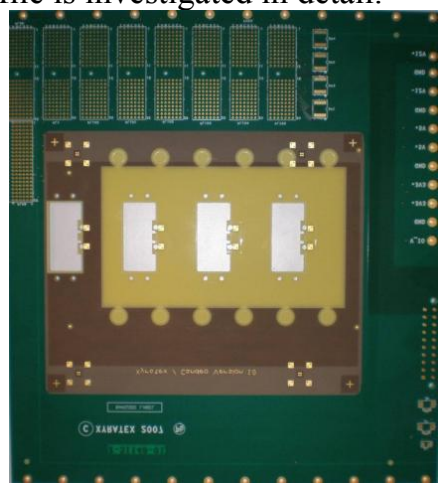

Figure 1. Optical PCB board waveguide interconnect. The white rectangles are the cut-out connector sites where the daughter boards are plugged into the backplane.

The cutting parameters are also optimized to achieve the best surface quality after cutting. Without applying any modification, the end facet roughness of the waveguide samples were scanned using atomic force microscopy (AFM) to measure and compare the surface profiles. In this paper, the results are presented in terms of roughness variation and chip load. Chip load is a measurement of the removed material by a cutting edge during a cut using a router[7]. Chip load is the ratio of the Feed rate/ (rotation speed in RPM $\times$ number of flutes). Feed rate is the translation speed and flutes are the cutting edges on the router. The optical loss was also measured and the results are reported.

\section{WAVEGUIDE END FACET CUT WITH DICING SAW AND MILLING ROUTER}

\subsection{Waveguide End Facets Cut with a Dicing Saw}

A dicing saw is commonly used by engineers to cut waveguides and the end facet roughness depends upon the dicing saw grit size and rotation and the translation speed of the cutter [8;9]. Figure 2 shows the end facet surface profile of a waveguide cut by a Disco DAD320 dicing machine (30,000 rpm, cutting speed $=0.6 \mathrm{~m} / \mathrm{min}$, diamond particle size on the resin blade $30 \mu \mathrm{m}$ ). As indicated in Figure 2, there are some lines or grooves on the end facet waveguide cross section surface and the AFM results show a roughness with standard deviation of $66.3 \pm 10 \mathrm{~nm}$; this magnitude of roughness will be compared with the milling methods later in this chapter (software was used to calculate the standard deviation of the roughness from the roughness data measured by the AFM machine
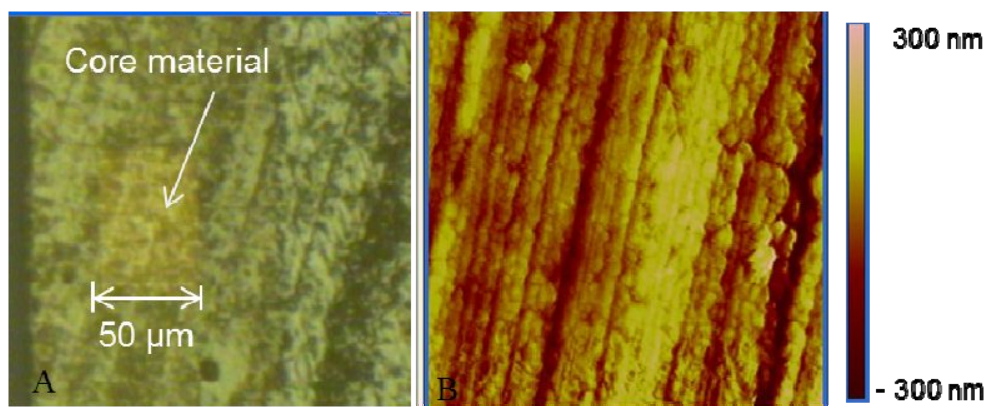

Figure 2. The roughness profile of the surface of a waveguide cut by a Disco DAD320 dicing saw with 30,000 rpm, cutting speed = $0.6 \mathrm{~m} / \mathrm{min}$. A: picture taken with an optical microscope, B: $30 \mu \mathrm{m} \times 30 \mu \mathrm{m}$ area of the core surface scanned by an AFM 


\subsection{Waveguide End Facets Cut with a Milling Router}

The use of a milling router to cut the board was considered and tried. It is easy to apply and control any cut by CNC machines and any range/size of cuts and shaped apertures are possible without causing any damage to the other part of the OPCB board. Milling routers have been used for cutting polymer waveguides [10;11]. However, the surface profile left after milling polymer waveguides has not been previously investigated in the backplane systems and it is essential to have the information on the roughness produced and the surface profile after cutting [12].

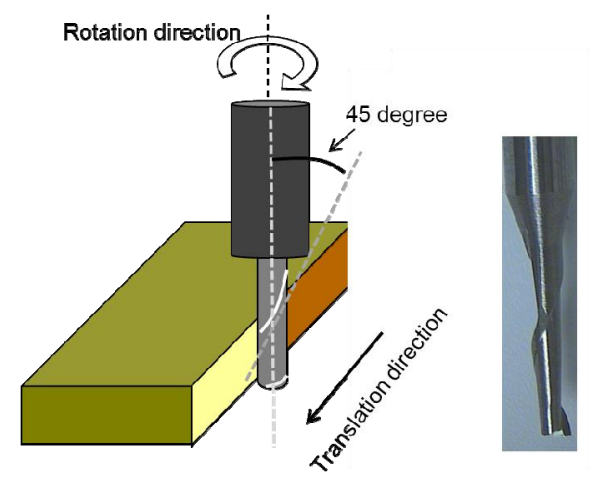

Figure 3. Schematic diagram of a one flute router and the cutting procedure used for the roughness investigation (from Lamar UK Ltd)

Three different router cutters with different numbers of cutting edges (flutes) were chosen to determine the most suitable for giving the end facet the smoothest surface. This is new research and no information is available for using flute routers to cut polymer waveguides. The waveguides were photo-lithographically manufactured acrylate waveguides and were cut by the PCB manufacturing company Stevenage Circuit in order to obtain realistic results from an actual PCB manufacturer. A silicon carbide $1.6 \mathrm{~mm}$ diameter flute router supplied by Lamar UK Ltd was used with a CNC milling machine, RLG 615 Drill/Router from Ernst Wessel Machinebau Gmbh, (Figure 3). The surface profiles of the samples were investigated using an optical microscope and AFM.

\subsection{Experiment results}

The experiments were started by making some samples to find in which way the waveguide should be placed in the CNC machine. There are two ways: first the waveguide (upper cladding) is facing up and secondly the copper/FR4 is facing up. Both of these options were tried and the first point was noted, which can be observed in Figure 4, is that when the waveguide is on the top surface during routing, the end of the waveguide is broken rather than being cut. Figure 4 which is the end of the waveguide after cutting, shows that the end of the waveguide is not flat but has been broken when the router is inserted from waveguide side (part A).

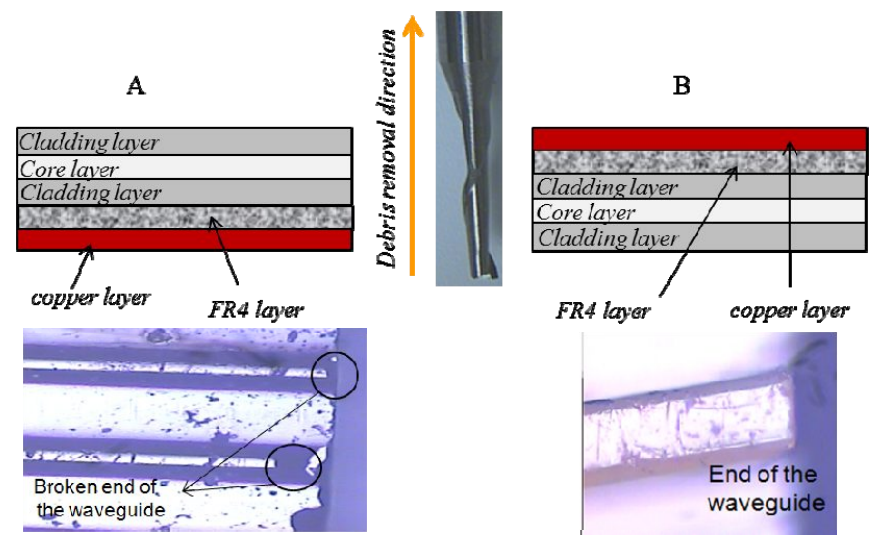

Figure 4. The end of the waveguide after cutting with the router entering the sample from A: waveguide and B: Copper/FR4 side 
When the waveguide is facing up (part A in Figure 4), there is only one layer of cladding on top of the core material and rotating the router pulls the layers upward and causes delamination and the broken end, however, when the waveguide is facing down (part B in Figure 4) during milling (the Copper/ FR4 layer is on the top in this situation), the force from the router on the waveguide tends to push the waveguide towards the surface on which it has been fabricated during cutting. Also there are more layers on top of the core material as a protection, namely, the lower cladding, FR4 and a copper layer, so we observed that, in this arrangement, the end facet was cut cleanly and not damaged.

It was necessary to determine the effect of the number of router cutter flutes on the surface finish. The shape of the router and the numbers of flutes on the router has been reported to affect other surfaces such as metal or plastic material cut by a milling machine (this information was provided by Stevenage Circuits Ltd.). Therefore, to find the effect of the number of flutes on the polymer waveguide surface quality, three cutters with different numbers of flutes, but with the same flute properties (material, flute angle, flute direction, etc.) were chosen and used to cut the waveguide samples. Figure 5 shows the surface profile of the waveguide cut by cutters with different numbers of flutes. The pictures were taken by using a Nomarski microscope and the waveguides back-illuminated. The yellow part is the core material of the waveguide surrounded by the cladding material.
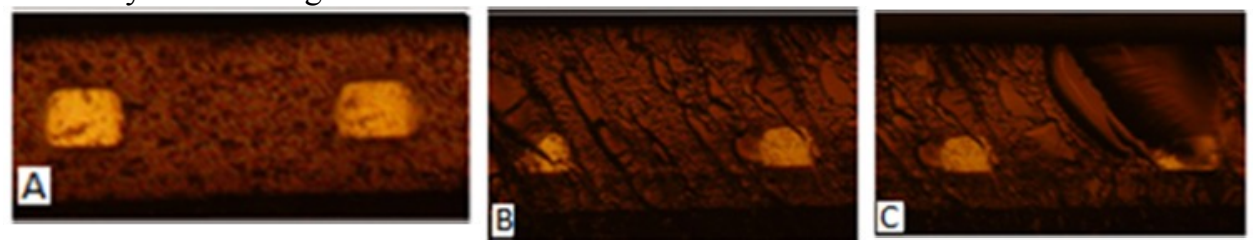

Figure 5. The surface profile of the waveguide cut by A: a one-flute router cutter $(\sigma=183 \mathrm{~nm}), \mathrm{B}$ : a two-flute cutter $(\sigma=540 \mathrm{~nm}), \mathrm{C}$ : Three-flute cutter $(\sigma=900 \mathrm{~nm})$.

As shown in Figure 5 there are some cracks and grooves in the samples cut by the two and three-flute router cutters.

Figure 6 indicates that roughness increases with number of flutes on the router. The three-flute router cutter leaves a very rough surface (standard deviation, $\sigma$, of $911 \pm 285 \mathrm{~nm}$ ). Some groove or gouge-shape structures could be observed on the surface cut by a three-flute cutter. The one-flute cutter gives a better and smoother surface (standard deviation, $\sigma$, of 183 $\pm 13 \mathrm{~nm}$ ). The AFM had to be controlled (by moving the tip up and down) manually for the samples cut by the threeflute router as the roughness was too high to be followed by the AFM tip. Moreover, a larger area, more than $30 \mu \mathrm{m} \times 30$ $\mu \mathrm{m}$, of the waveguide surface could not be scanned by the AFM as there was a large groove which caused the AFM machine to show errors. It was observed that the samples cut by the two and three-flute routers were too rough for the AFM (we were able to take a few scans by choosing a smoother area.

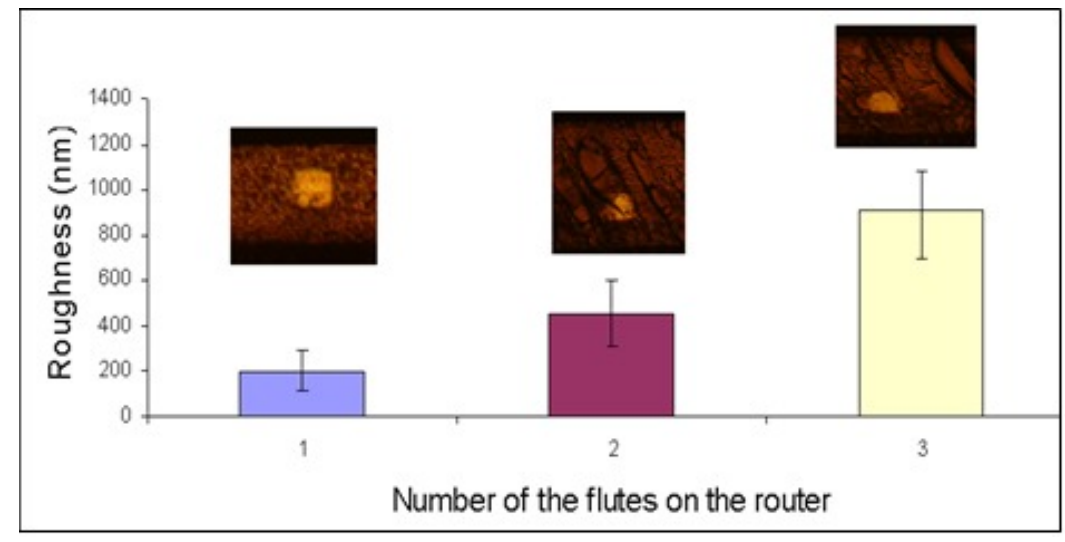

Figure 6. A sample of the Standard deviation of roughnesses of the surfaces cut by router cutters with different numbers of flutes. $15000 \mathrm{rpm}$, cutting speed $=0.25 \mathrm{~m} / \mathrm{min}$

The optimum spindle and cutting speeds required for minimum surface roughness were determined. Table 1 shows the standard deviation of the roughness of the various surfaces cut at different cutter rotation and translation speeds.

Figure 7 shows a 3D plot of the end facet roughness data presented in Table 1. The graph shows a minimum area like a diagonal valley in the middle of the plot. This is the blue area in the contour plot in Figure 7. This means that to achieve 
a surface with lower magnitude of roughness, the translation speed should be increased if there is an increase in the rotation speed. In other words, small rotation speeds should be used with small translation speed and vice versa.

Table 1. Standard deviation of the surface roughness (in nanometers) for surfaces cut with a one-flute router at different rotation and translation speeds. The unit of the roughness, chip load and translation speed are $\mathrm{nm}, \mu \mathrm{m}$ and $\mathrm{m} / \mathrm{min}$ respectively

\begin{tabular}{|c|c|c|c|c|c|}
\hline \multirow{2}{*}{\multicolumn{2}{|c|}{$\frac{\text { Rotation speed (RPM) }}{\underset{\mid}{\mid} \text { Translation Speed (m/min) }}$}} & \multirow{3}{*}{$\begin{array}{c}\mathbf{5 0 0 0} \\
389 \pm 61\end{array}$} & \multirow{3}{*}{$\begin{array}{c}15000 \\
310 \pm 12\end{array}$} & \multirow{3}{*}{$\begin{array}{c}34000 \\
384 \pm 10\end{array}$} & \multirow[t]{2}{*}{50000} \\
\hline & & & & & \\
\hline \multirow{2}{*}{0.10} & Roughness & & & & $342 \pm 23$ \\
\hline & Chip load & 20 & 6.6 & 2.9 & 2 \\
\hline \multirow{2}{*}{0.25} & Roughness & $376 \pm 30$ & $183 \pm 13$ & $358 \pm 12$ & $339 \pm 42$ \\
\hline & Chip load & 50 & 16 & 7.4 & 5 \\
\hline \multirow{2}{*}{0.50} & Roughness & $474 \pm 32$ & $381 \pm 18$ & $205 \pm 24$ & $410 \pm 15$ \\
\hline & Chip load & 100 & 33 & 15 & 10 \\
\hline \multirow{2}{*}{0.75} & Roughness & $434 \pm 54$ & $386 \pm 20$ & $407 \pm 43$ & $296 \pm 12$ \\
\hline & Chip load & 150 & 50 & 22 & 14 \\
\hline
\end{tabular}

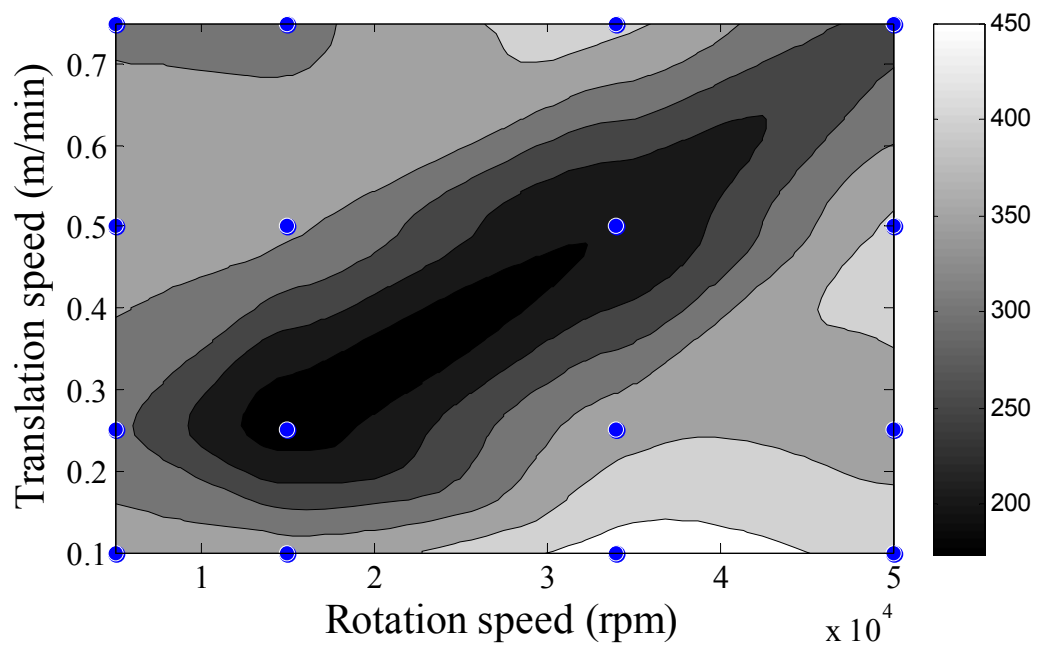

Figure 7. End facet roughness for different rotation and feed speeds. Blue indicates low roughness and red high roughness

In theory, higher translation speeds are possible with higher rotation speeds as the material is removed faster. However, results show that a greater rotational speed generates higher frictional heating between the router cutter and the sample which can melt the polymer and also create increased mechanical wear on the cutting edge. 


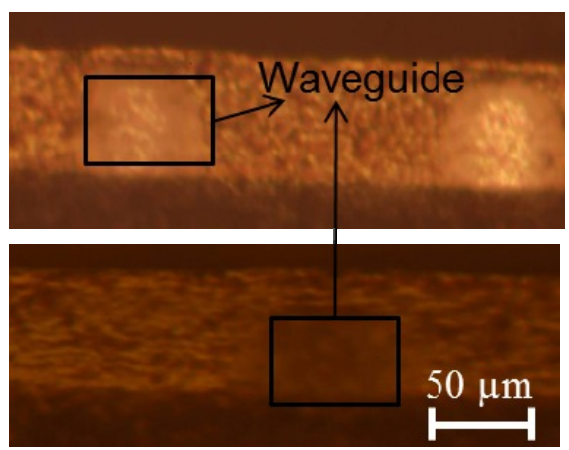

Figure 8. A photograph of the waveguide end facet taken by a Nomarski Optical microscope from a sample cut with rotation speed $=$ $34,000 \mathrm{rpm}$, translation speed $=0.75 \mathrm{~m} / \mathrm{min}$

Figure 8 shows the end facet of a waveguide sample that was cut at 34,000 rpm. The waveguide sample was back illuminated to distinguish the core materials but the waveguides were either blurred or blocked as we could not observe a clear waveguide core. It appears as if the waveguide surface has been covered with another layer. It is not dust or small swarf particles that can be cleaned off the surface. The rotation speed was increased to 70,000 rpm but with no improvement in the roughness

\section{COMPARING THE WAVEGUIDE SURFACE ROUGHNESS WITH THAT OF A SILICON}

Having determined the best rotation and feed speeds for cutting the waveguides, the three different surfaces roughnesses in Figure 9 were compared after milling. The roughness of a silicon wafer was considered as an ideal polished surface for comparison of the waveguide roughness cut by a diamond saw and the same waveguide cut by the one flute router.

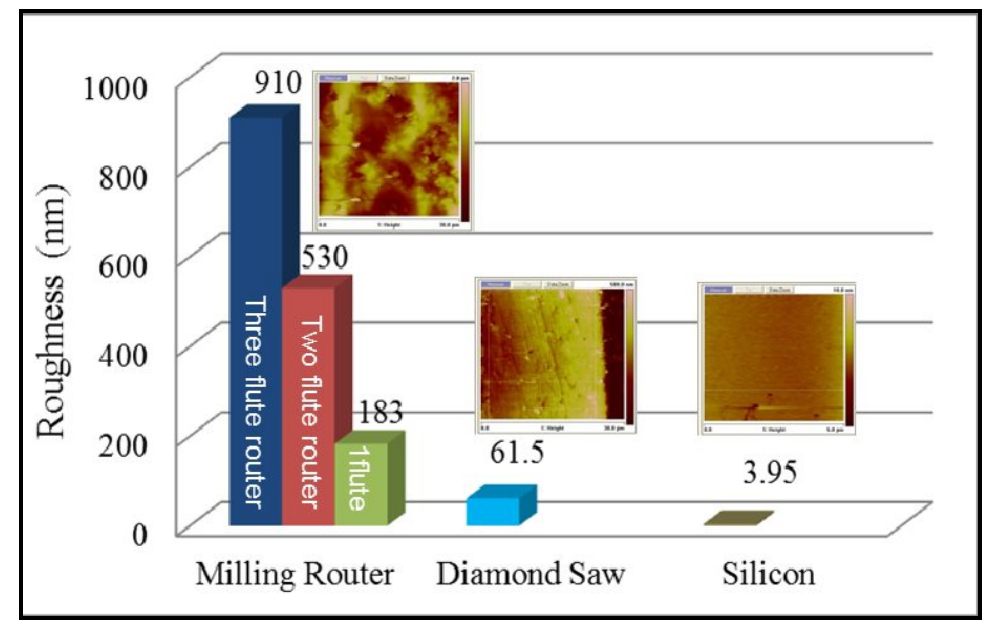

Figure 9: Roughness of the surfaces of silicon and waveguides cut by dicing saw and routers with different number of cutting edges. An AFM picture is shown for each surface for comparison of the surface profile. The number on top of each bar is the standard deviation of the roughness in $\mathrm{nm}$ for that sample.

\subsection{Optical Input/Output Coupling Loss due to Roughness of Waveguide End}

To discover the relation between the roughness magnitude and optical loss, the input/output optical loss due to the different end facet roughness is measured. The length of the samples was $10 \mathrm{~mm}$ in order to fit under the AFM tip and a multimode fibre coupled VCSEL with $853 \mathrm{~nm}$ wavelength was used as the source. The receiver was a photo-detector with a circular aperture of $70 \mu \mathrm{m}$ and the waveguides' cross section was $50 \mu \mathrm{m} \times 50 \mu \mathrm{m}$. The measurements were performed based on the experimental method described in ref [13]. An index matching fluid was also used to reduce the coupling loss without reducing the physical roughness. The liquid penetrates between the microstructure of the roughness and fills the space between waveguide surface fluctuations. An index matching fluid with $n=1.5694 \pm$ 0.0005, made by Cargille Laboratories (code: LCABK), was applied to the multimode fibre and the input facet of the 
waveguide. It was also added between the output facet roughnesses of the waveguide. Figure 10 shows the results of the measurement.

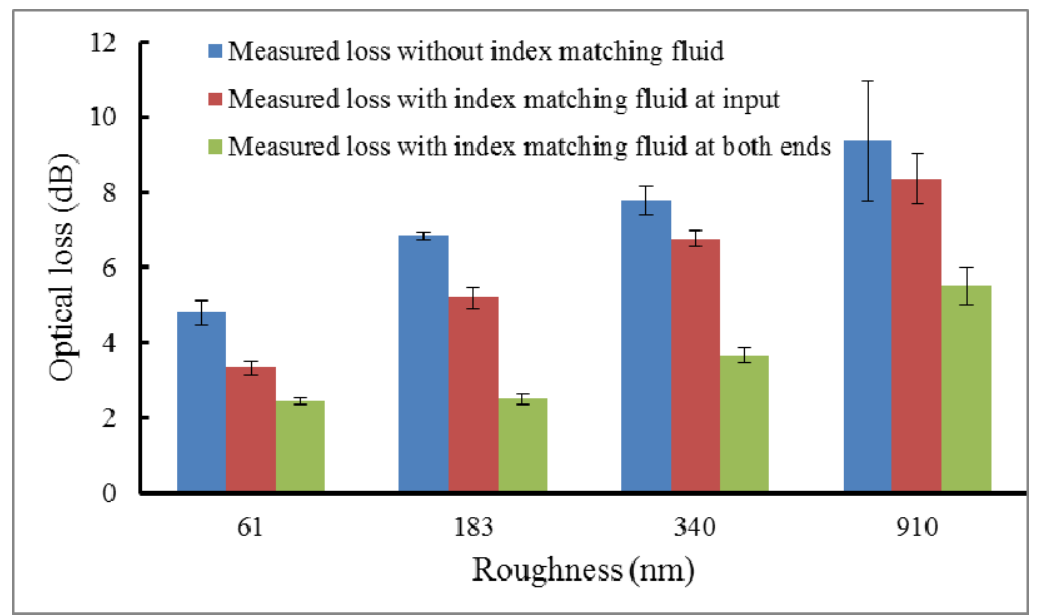

Figure 10. Optical loss of one crossing as a function of the angle of the crossing for a calculation using ray tracing compared to the experimental result. The input source has an $\mathrm{NA}=0.22$

Figure 10 shows the measured loss for four different magnitudes of end facet roughness. To be precise, the measured loss is the insertion loss which is the coupling loss plus the optical loss due to propagation loss which includes losses due to sidewall roughness scattering and due to material absorption; however, as the waveguide samples are $1 \mathrm{~cm}$ long and the propagation loss is $0.05 \mathrm{~dB} / \mathrm{cm}$ (has been measured previously in our research group), the insertion loss can be ignored compared to the coupling loss. The samples were four waveguides fabricated on the same wafer. In fact it was a large $(10 \mathrm{~cm})$ straight waveguide which was cut into several pieces. Both ends of each sample were cut with the same method; therefore the roughness quoted on the horizontal axis is for both ends.

The last section of this paper describes a new method that has been developed for removing the end facet roughness permanently; this is the use of waveguide core material to form a thin layer at the end facet of the waveguides to reduce its roughness. This behaves like an index matching fluid but without its drawbacks. The procedure implemented is shown in Figure 11 Firstly, a small amount of liquid core polymer is applied to one end of the optical layer. Secondly a silicone lubricant aerosol release agent (CP1051 produced by CRC Industries UK Ltd) covers one side of a glass slide (made by Menzel-Glaser) and is pushed (about $400 \mathrm{~Pa}$ pressure) against the waveguide end facet. The surface roughness of the microscope slide is $9 \pm 3 \mathrm{~nm}$ (measured by AFM). The slide was used for two reasons; first to achieve a flat surface at the end of the waveguide and second, to be transparent and to make sure the added core material does not go beyond the cut waveguide. Ultraviolet (UV) light was then used to cure the polymer through the glass slide which was then removed. The coupling loss was measured and the process repeated for the other side of the waveguide facet. 


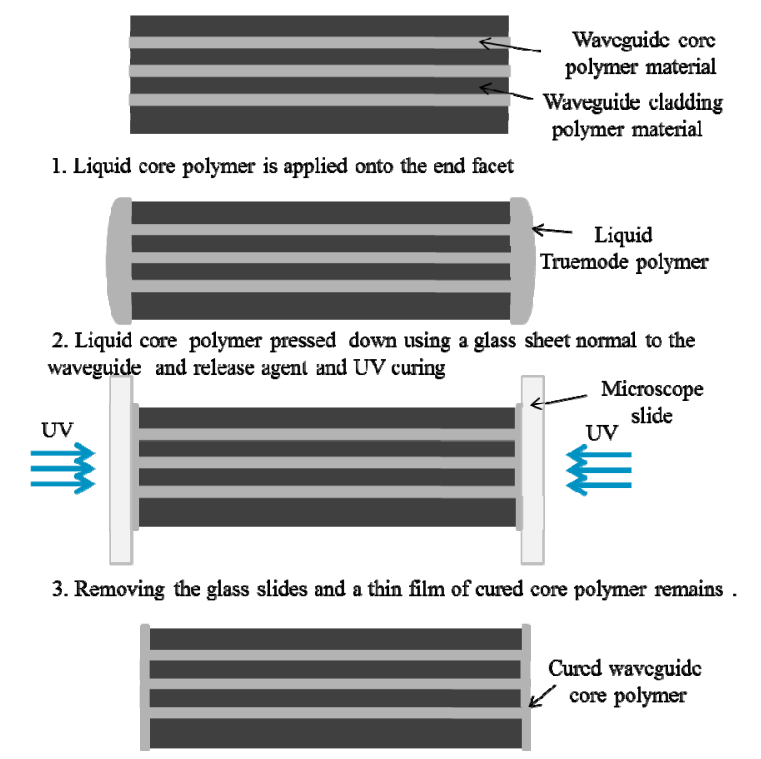

Figure 11: Procedure for waveguide end facet smoothing technique using core material.

Nine waveguides of different lengths in an OPCB board [14] were measured before and after applying the refractive index matching fluid. Figure 12 shows the measurements of the insertion loss in comparison with optical loss measurements after applying the core material to the end of the waveguide. The graph indicates that the loss is reduced by the same or a greater extent than by using the index matching fluid method.

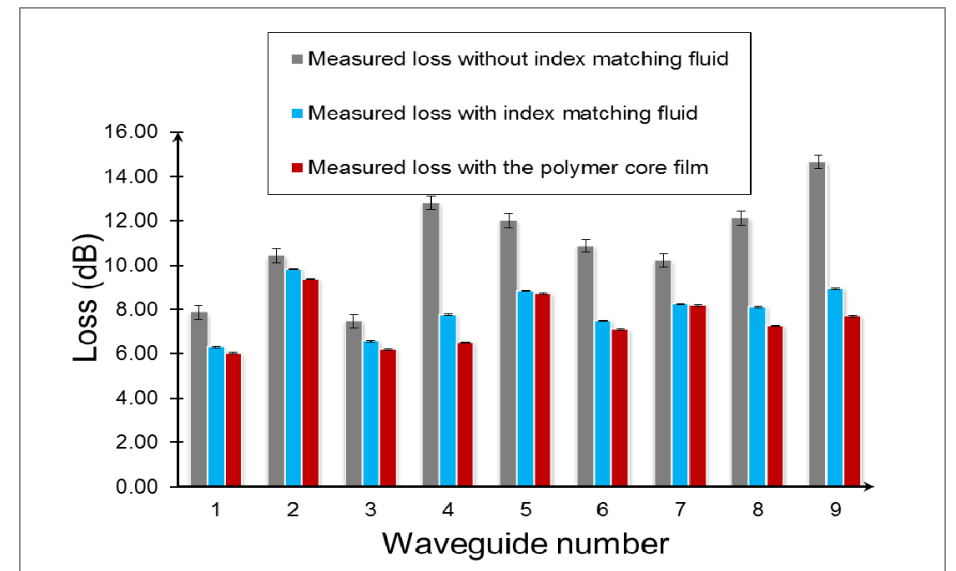

Figure 12: The measured loss for different values of end facet roughness. The improvement of the coupling loss after applying index matching fluid averages $2.23 \pm 1.2 \mathrm{~dB}$, and after applying acrylate is $2.60 \pm 1.3 \mathrm{~dB}$.

\subsection{Conclusions:}

In this paper, detailed characterisation studies of the end facet roughness of polymer waveguides suitable for use in board-level optical interconnects are presented and a novel method of reducing the related optical coupling losses is proposed. The paper addresses some of the practical issues related to the adoption of this technology in real-world systems. The end facet roughness of multimode polyacrylate waveguides fabricated on FR4 PCBs, when cut at right angles to their optical axis by milling routers for optical butt-coupling connectors is compared with that resulting from dicing saws and polishing. The RMS surface roughnesses of polyacrylate waveguide end facets on FR4 PCB, due to milling routing at a range of rotation speeds and translation speeds are compared. One-flute routers gave significantly less rough surfaces than two or three-flute routers. The best results were achieved for a one-flute router when the milling bit was inserted from the PCB side of the board and the research optimized the milling parameters to minimize the optical loss. For the first time, to our knowledge, the waveguide optical insertion loss is shown to be linearly proportional 
to the ratio of the waveguide core end facet RMS roughness to its autocorrelation length. A new fabrication technique giving a more robust end facet for use in commercial products is proposed and demonstrated which reduces the insertion loss.

\section{REFERENCES}

[1] I. Papakonstantinou and David R.Selviah, "Analysis design and measurment of guided wave optical backplane interconnection." PhD thesis University College London, 2007.

[2] D. R. Selviah, A. C. Walker, D. A. Hutt, K. Wang, and H. Baghsiahi, "Integrated Optical and Electronic Interconnect PCB Manufacturing Research," Circuit World, vol. 36, no. 2, pp. 5-19, 2010.

[3] I. Papakonstantinou, D. R. Selviah, K. Wang, R. A. Pitwon, K. Hopkins, and D. Milward, "Optical 8-channel, $10 \mathrm{~Gb} / \mathrm{s}$ MT pluggable connector alignment technology for precision coupling of laser and photodiode arrays to polymer waveguide arrays for optical board-to-board interconnects," Electronic Components and Technology Conference, 2008. ECTC 2008. 58th, pp. 1769-1775, 2008.

[4] H. Baghsiahi, D. R. Selviah, G. Yu, K. Wang, M. Yau, and F. A. Fernandez, "Photolithographically manufactured acrylate multimode optical waveguide translation and rotation misalignment tolerances," Proceedings- 2008 2nd Electronics System Integration Technology Conference, ESTC, pp. 617-621, 2008.

[5] B. Huang, J. Chen, and W. S. Jiang, "Effects of Surface Roughness on TE Modes in Rectangular Waveguide," Journal of Infrared Millimeter and Terahertz Waves, vol. 30, no. 7, pp. 717-726, 2009.

[6] R. Pitwon, K. Wang, J. Graham-Jones, I. Papakonstantinou, H. Baghsiahi, B. Offrein, R. Dangel, D. Milward, and D. Selviah, "FirstLight: Pluggable Optical Interconnect Technologies for Polymeric Electro-Optical Printed Circuit Boards in Data Centers," IEEE Journal of Lightwave Technology, vol. 30, no. 21, pp. 3316-3329, Nov. 2012.

[7] Vortex tool ltd, "Chip load chart and information," http://www.vortextool.com/images/chipLoadChart.pdf, 2011.

[8] M. Saillard and D. Maystre, "Scattering from Metallic and Dielectric Rough Surfaces," Journal of the Optical Society of America A-Optics Image Science and Vision, vol. 7, no. 6, pp. 982-990, 1990.

[9] S. Cvetkovic, C. Morsbach, and L. Rissing, "Ultra-precision dicing and wire sawing of silicon carbide (SiC)," Microelectronic Engineering, vol. 88, no. 8, pp. 2500-2504, 2011.

[10] M. Komatsu, "Machining Performance of A Router Bit in the Peripheral Milling of Wood .2. Effects of the Helix Angle of the Peripheral Cutting-Edge on the Cutting Force and Machined-Surface Roughness," Mokuzai Gakkaishi, vol. 40, no. 2, pp. 134-141, 1994.

[11] H. Kamata and T. Kanauchi, "Analysis of Machined Surfaces with Distal Image-Processing .1. Effect of Grain Angle in Numerical Control Router Machining," Mokuzai Gakkaishi, vol. 39, no. 11, pp. 1253-1258, 1993.

[12] M. E. Teitelbaum, R. Nair, D. J. O'Brien, E. D. Wetzel, and K. W. Goossen, "Cost-effective integration of plastic optical fiber and total internal reflection mirrors in printed circuit boards for parallel optical interconnects," Optical Engineering, vol. 49, no. 6, pp. 456-461, 2010.

[13] H. Baghsiahi, K. Wang, W. Kandulski, R. C. Pitwon, and D. R. Selviah, "Optical Waveguide End Facet Roughness and Optical Coupling Loss," Lightwave Technology, Journal of, vol. 31, no. 16, pp. 2659-2668, 2013. 
[14] K. Wang, D. R. Selviah, J. Papakonstantinou, G. Yu, H. Baghsiahi, and F. A. Fernandez, "Photolithographically manufactured acrylate polymer multimode optical waveguide loss design rules," Proceedings - 2008 2nd Electronics Systemintegration Technology Conference, ESTC, pp. 1251-1255, 2008. 